\title{
Eficácia e segurança do tramadol em indivíduos com ejaculação prematura: uma revisão baseada na evidência
}

Isabel Machado, ${ }^{1}$ Helena Machado, ${ }^{2}$ Sara Domingues ${ }^{2}$

\section{RESUMO}

Objetivos: A ejaculação prematura (EP) é a disfunção sexual masculina mais frequente e a sua prevalência ronda os $20-30 \%$. Nos últimos anos, várias alternativas farmacológicas têm sido usadas no tratamento da EP. Um dos tratamentos usados, on demand, que recentemente mostrou ser promissor no tratamento da EP primária é o tramadol. Contudo, há estudos que demonstram resultados inconsistentes relativamente ao seu uso. Assim, este estudo teve como objetivo rever a evidência mais atual sobre a eficácia e a segurança do uso do tramadol no tratamento da EP.

Fontes de dados: National Guideline Clearing House, NICE, Canadian Medical Association Practice Guidelines, DARE, Cochrane, PubMed e TRIP Database.

Métodos de revisão: Pesquisa de metanálises (MA), revisões sistemáticas (RS), estudos observacionais e normas de orientação clínica/guidelines baseadas na evidência (NOC), publicadas em português, espanhol e inglês, utilizando os termos MeSH tramadol e premature ejaculation. Foi utilizada a escala Strength of Recommendation Taxonomy (SORT) da American Academy of Family Physicians para atribuição dos níveis de evidência e forças de recomendação.

Resultados: Foram obtidos 56 artigos e, destes, sete cumpriram os critérios de inclusão: duas NOC, quatro MA e uma RS. A maioria dos estudos revelou que o uso do tramadol é efetivo relativamente ao placebo, mas não quando comparado com a paroxetina. Porém, as diferentes metodologias aplicadas nos vários estudos contribuíram para a heterogeneidade dos resultados, diminuindo a firmeza das conclusões obtidas.

Conclusão: Perante a evidência disponível, esta revisão permitiu-nos concluir que a utilização do tramadol no tratamento da EP apresenta evidência limitada (Força Recomendação B). Serão necessários estudos futuros de elevada qualidade, envolvendo um maior número de indivíduos e com critérios de inclusão semelhantes e uniformização dos outcomes avaliados, de forma a obter conclusões sustentadas sobre o uso de tramadol a longo prazo.

Palavras-chave: Tramadol; Ejaculação prematura

\section{INTRODUÇÃO}

A ejaculação prematura (EP) é a disfunção sexual masculina mais frequente e estima-se que a sua prevalência ronde os $20-30 \% .{ }^{1} \mathrm{Em}$ contraste à disfunção erétil, que geralmente ocorre em homens mais velhos, a EP é vista em homens adultos de todas as idades. ${ }^{2}$ Várias definições têm sido adotadas para a EP; contudo, uma definição universal-

1. Médica interna de Medicina Geral e Familiar. USF do Minho, ACeS Cávado I - Braga 2. Médica interna de Medicina Geral e Familiar. USF Pró-Saúde, ACeS Cávado II Gerês/Cabreira mente aceite ainda tem que ser estabelecida. Em 2008, a Sociedade Internacional de Medicina Sexual (ISSM) adotou uma definição baseada na evidência científica, sendo aquela que tem criado mais consenso na comunidade urológica: "ejaculação prematura é uma disfunção sexual masculina caracterizada pela ejaculação que ocorre, geralmente ou sempre, antes da penetração vaginal ou até um minuto após, associada à incapacidade de retardar esta ejaculação, causando consequências pessoais, como incómodo, angústia, frustração e aversão à intimidade sexual". ${ }^{3}$ Baseada nestes cri- 
térios, a medição do tempo de latência ejaculatória intravaginal (TLEI) foi considerada como elemento-chave na avaliação da eficácia dos diferentes agentes usados no tratamento da EP. A EP pode ser classificada como permanente (primária) ou adquirida (secundária). ${ }^{4} \mathrm{~A}$ EP primária é uma condição que está presente desde o início da maturidade sexual e permanece durante a vida, enquanto a EP secundária é caracterizada por um início súbito ou gradual, que se desenvolve após um intervalo de função sexual normal. ${ }^{4}$

A ejaculação é regulada por numerosos mecanismos. A exata etiologia da EP é ainda desconhecida e pensa-se que resulte da combinação de componentes psicológicos e/ou neurobiológicos, hipersensibilidade peniana e disfunção dos recetores da serotonina. ${ }^{5-7}$

Tratamentos farmacológicos para a EP incluem analgésicos tópicos e terapêuticas sistémicas como os inibidores seletivos da recaptação da serotonina (SSRIs) e os inibidores da fosfodiesterase-5 (PDE-5), mas o seu uso para a EP é off-label. Apenas a dapoxetina, um novo SSRI, foi aprovado para o uso, on demand, no tratamento da EP em determinados países europeus, incluindo Portugal. ${ }^{8}$

Um dos tratamentos usados, on demand, que recentemente mostrou ser promissor no tratamento da EP primária é o tramadol. ${ }^{9} \mathrm{O}$ tramadol é um analgésico opióide sintético, de ação central, que pode atrasar a ejaculação através de dois mecanismos de ação distintos: estimulação do recetor $\mu$-opióide e inibição da recaptação da noradrenalina e da 5-hidroxitriptamina (5-HT)..$^{10-11}$ Vários estudos têm mostrado que o tramadol é eficaz e seguro no tratamento da $\mathrm{EP}$, através do aumento do TLEI. ${ }^{9-10,12-13}$ Contudo, outros estudos demonstraram resultados inconsistentes relativamente ao uso do tramadol na EP, questionando a segurança do tramadol e alertando para os seus efeitos laterais severos. $^{14}$

Assim, esta revisão teve como objetivo rever a evidência mais atual sobre a eficácia e a segurança do uso do tramadol no tratamento da EP.

\section{MÉTODOS}

Foi realizada uma pesquisa bibliográfica, em abril de 2016, de metanálises (MA), revisões sistemáticas (RS), estudos observacionais e normas de orientação clínica/guidelines baseadas na evidência (NOC), inde- xados nas bases de dados da National Guideline Clearing House, NICE, Canadian Medical Association Practice Guidelines, DARE, Cochrane, PubMed e TRIP Database, de artigos nas línguas portuguesa, espanhola e inglesa, sem limite temporal. Foram utilizados os termos MeSH tramadol e premature ejaculation.

Foram incluídos na revisão artigos que cumprissem os seguintes critérios: a) População: indivíduos do sexo masculino com idade igual ou superior a 18 anos com EP; b) Intervenção: tramadol; c) Comparação: placebo ou outros tratamentos; d) Outcome: eficácia, através do aumento do TLEI e segurança, através da incidência de efeitos adversos.

Foi utilizada a escala Strength of Recommendation Taxonomy (SORT) da American Academy of Family Physicians na avaliação dos estudos e atribuição dos níveis de evidência (NE) e forças de recomendação (FR).

\section{RESULTADOS}

Foram obtidos 56 artigos, tendo sido selecionadas duas NOC, quatro MA e uma RS. O processo de seleção dos estudos incluídos está representado no fluxograma da Figura 1.

As NOC incluídas neste artigo de revisão são contraditórias (Quadro I). Segundo as guidelines da International Society for Sexual Medicine's, ${ }^{15}$ publicadas em 2010, o tratamento da EP com tramadol não é recomendado (NE 2d). Mais recentemente, as guidelines da European Association of Urology, ${ }^{16}$ publicadas em 2013, referem que o tramadol demonstrou ter um efeito benéfico moderado, com uma eficácia semelhante à dapoxetina. Os autores concluíram que o uso do tramadol, on-demand, pode ser usado no tratamento da EP (FR B). Contudo, referem que a eficácia e a tolerabilidade devem ser confirmadas com estudos que incluam mais indivíduos e que avaliem a eficácia e a segurança a longo prazo.

A MA de Wu e colaboradores, publicada em 2012 (Quadro II), teve como objetivo principal avaliar a eficácia e a segurança do tramadol no tratamento da EP. ${ }^{17}$ Os autores avaliaram separadamente a eficácia (através da medição do TLEI) e a segurança (através da frequência de efeitos adversos). Relativamente à eficácia, os autores compararam dois grupos: tramadol versus placebo e tramadol versus paroxetina. Para o primeiro grupo incluíram cinco ensaios clínicos que envolveram 


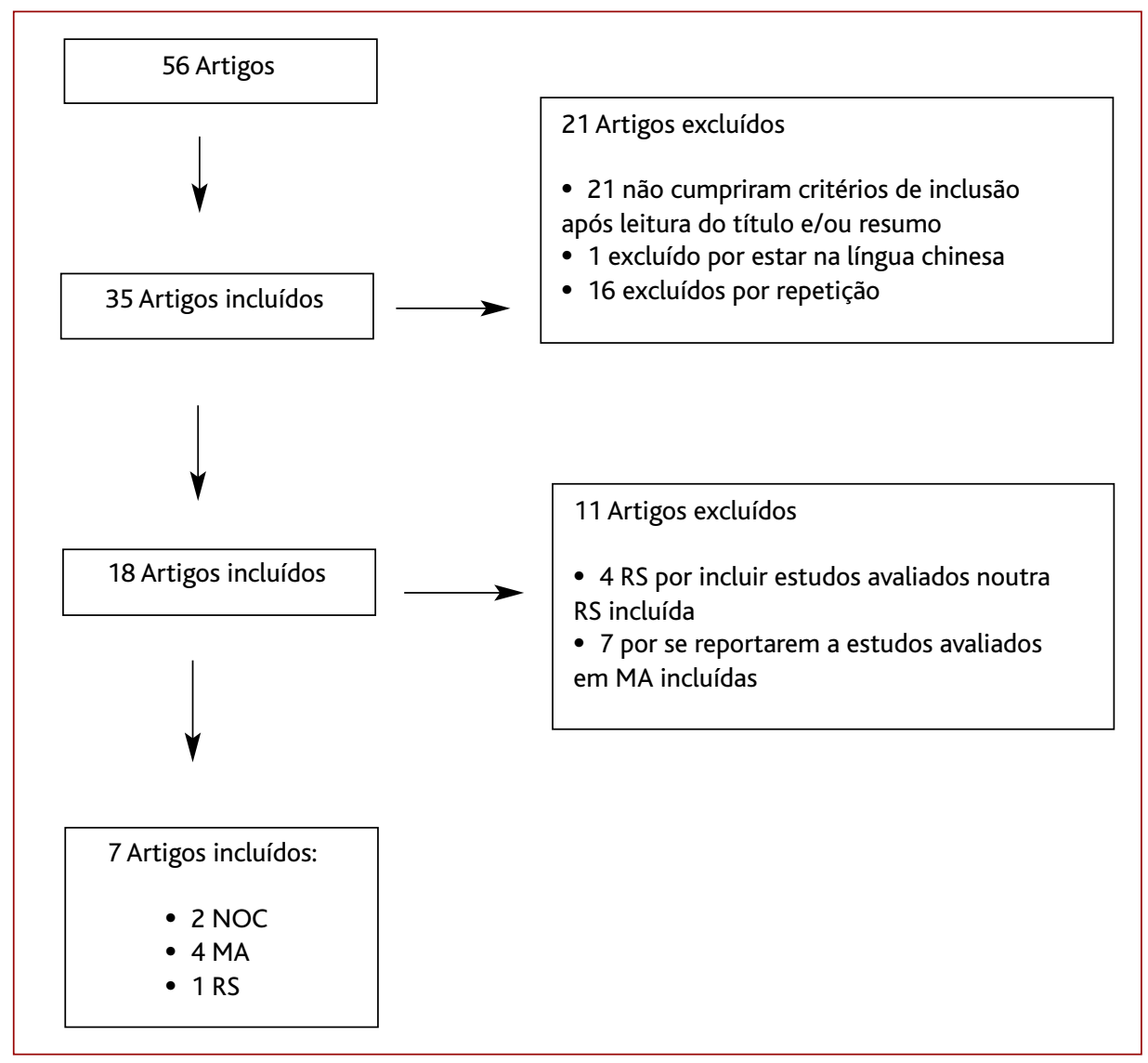

Figura 1. Esquema de seleção dos estudos.

Legenda: $M A=$ Metanálise, $R S=$ Revisão sistemática, NOC=Norma de orientação clínica.

ram 228 casais (114 para tramadol e para paroxetina). Os autores concluíram que não houve diferenças estatisticamente significativas entre $o$ tramadol e a paroxetina. Para avaliar os efeitos adversos compararam o tramadol e o placebo, tendo incluído três ensaios clínicos. Verificaram que o tramadol teve uma taxa mais elevada de efeitos adversos, sendo os mais frequentes a sonolência e o desconforto gastrointestinal.

A MA de Yang e colaboradores, publicada em 2013 (Quadro II), incluiu cinco ensaios clínicos que envolveram 715 indivíduos com EP. ${ }^{18}$ Compararam o uso de tramadol (361 indivíduos) versus placebo (354 indivíduos) relativamente à eficácia e segurança. Concluíram que o tramadol (nas doses de 25,50 e $62 \mathrm{mg}$ ) aumentou significativamente o TLEI e, embora os efeitos adversos tenham sido ligeiros a moderados, foram significativamente maiores relativamente ao grupo controlo.

A MA de Castiglione e colaboradores,

1.129 casais (567 para o tramadol e 562 para o placebo) que foram subdivididos, consoante as diferentes doses de tramadol recebidas ( $25 \mathrm{mg}, 50 \mathrm{mg}, 62 \mathrm{mg}$ e $89 \mathrm{mg}$ ). Os autores verificaram que o efeito do tramadol é estatisticamente superior ao placebo no prolongamento do TLEI, independentemente da dose. Para o segundo grupo foram incluídos dois ensaios clínicos que envolve- publicada em 2016 (Quadro II), incluiu três ensaios clínicos que compararam o uso do tramadol (nas doses de $50 \mathrm{mg}$, $62 \mathrm{mg}$, $89 \mathrm{mg}$ e $100 \mathrm{mg}$ ) versus placebo e verificaram que o tramadol provoca um prolongamento estatisticamente significativo do TLEI, comparativamente ao placebo. ${ }^{19}$ Contudo, os autores afirmam que a eficácia do tramadol permanece ainda incerta devi- 


\begin{tabular}{|c|c|c|c|c|}
\hline Referência & Estudos incluídos & Intervenção & Resultados & NE \\
\hline $\begin{array}{l}\text { Wu T, et al } \\
(2012) \\
\text { China }\end{array}$ & $\begin{array}{l}7 \text { Ensaios clínicos } \\
\text { (3 EAC e } 4 \text { ECNA) } \\
\text { Follow-up } 3 \text { a } 12 \\
\text { semanas }\end{array}$ & $\begin{array}{l}\text { Tramadol vs. Placebo } \\
\text { Outcomes: Eficácia } \\
\text { (2 EAC + } 3 \text { ECNA) } \\
\text { e segurança } \\
\text { (2 EAC + } 4 \text { ECNA) } \\
\text { Tramadol vs. } \\
\text { Paroxetina } \\
\text { Outcomes: Eficácia } \\
\text { (2 ECNA) }\end{array}$ & $\begin{array}{l}\text { O efeito do tramadol é estatisticamente superior ao placebo em } \\
\text { prolongar o TLEI no tratamento da EP, independentemente da } \\
\text { dose (SMD } 1,20 ; \text { IC } 95 \%: 1,12-4,47 ; P=0,001 \text { ) } \\
\text { Comparando com o placebo, o tramadol teve uma taxa mais } \\
\text { elevada de efeitos adversos (OR } 2,89 ; \text { IC } 95 \%: 1,88-4,43 ; \\
P<0,0001 \text { ) } \\
\text { Não houve diferenças estatisticamente significativas entre o uso } \\
\text { de tramadol e paroxetina no tratamento da EP (SMD- } 0,44 ; \text { IC } 95 \% \text { : } \\
-5,07-4,18 ; P=0,85 \text { ) }\end{array}$ & 2 \\
\hline $\begin{array}{l}\text { Yang L, et al } \\
\text { (2013) } \\
\text { China }\end{array}$ & $\begin{array}{l}5 \text { EAC } \\
\text { Follow-up? }\end{array}$ & $\begin{array}{l}\text { Tramadol vs. Placebo } \\
\text { Outcomes: Eficácia e } \\
\text { segurança ( } 5 \text { EAC) }\end{array}$ & $\begin{array}{l}\text { O uso de tramadol aumentou significativamente os valores de } \\
\text { TLEI, relativamente ao uso de placebo (SMD } 3,51 ; \text { IC95\%: } \\
2,14-4,88 ; P<0,00001 \text { ) } \\
\text { Os efeitos adversos no grupo medicado com tramadol foram } \\
\text { significativamente maiores do que no grupo controlo (RR } 3,55 \text {; } \\
\text { IC95\%: } 1,34-9,40 ; P=0,01 \text { ) }\end{array}$ & 1 \\
\hline $\begin{array}{l}\text { Castiglione } \\
\text { F, et al } \\
\text { (2016) } \\
\text { França }\end{array}$ & $\begin{array}{l}22 \text { EAC } \\
\text { Follow-up } 4 \text { a } 12 \\
\text { semanas }\end{array}$ & $\begin{array}{l}\text { Tramadol vs. Placebo } \\
\text { Outcomes: Eficácia } \\
\text { (3 EAC) }\end{array}$ & $\begin{array}{l}\text { O tratamento da EP com tramadol tem um efeito estatisticamente } \\
\text { significativo em prolongar o TLEI, comparativamente ao placebo } \\
\text { (SMD 2,02; IC95\%: 0,80-3,25; } \mathrm{P}<0,00001 \text { ) }\end{array}$ & 2 \\
\hline $\begin{array}{l}\text { James } M \text {, } \\
\text { et al (2015) } \\
\text { Reino } \\
\text { Unido }\end{array}$ & $\begin{array}{l}8 \text { EAC } \\
\text { Follow-up } 4 \text { a } 6 \\
\text { meses }\end{array}$ & $\begin{array}{l}\text { Tramadol vs. Sildenafil } \\
\text { Outcome: Eficácia } \\
\text { (1 EAC) } \\
\text { Tramadol (50mg) + } \\
\text { terapia } \\
\text { comportamental vs. } \\
\text { terapia } \\
\text { comportamental } \\
\text { Outcome: Eficácia e } \\
\text { segurança (1 EAC) } \\
\text { Tramadol vs Lidocaína } \\
\text { Outcome: Eficácia } \\
\text { (1 EAC) }\end{array}$ & $\begin{array}{l}\text { Após } 8 \text { a } 12 \text { semanas de tratamento, o efeito do tramadol no } \\
\text { prolongamento do TLEI é significativamente superior (SMD 1,24; } \\
\text { IC95\%: } 0,52-1,92 ; P=0,009 \text { ) } \\
\text { Após } 8 \text { a } 12 \text { semanas de tratamento, o tramadol apresenta } \\
\text { significativamente mais risco de efeitos adversos (RR } 2,27 ; \text { IC95\%: } \\
\text { 1,45-3,57; } P=0,0004) \\
\text { Após } 4 \text { semanas de tratamento, o efeito do tramadol no } \\
\text { prolongamento do TLEl é significativamente superior (MD 2,01; } \\
\text { IC95\%: } 1,21-2,87 ; P<0,00001 \text { ) } \\
\text { Após } 12 \text { semanas de follow- } u p \text {, o tratamento combinado do } \\
\text { tramadol com a terapia comportamental está associado a um } \\
\text { prolongamento significativamente maior do TLEI (MD 1,65; } \\
\text { IC95\%: } 0,30-3,00 ; P=0,02 \text { ) } \\
\text { Após } 12 \text { semanas de follow- } u p \text {, o tratamento combinado do } \\
\text { tramadol com a terapia comportamental apresenta maior risco de } \\
\text { efeitos adversos (RR } 21 ; \text { IC } 95 \%: 1,28-345,410 ; P=0,03 \text { ) } \\
\text { Após } 4 \text { semanas de tratamento, o efeito do tramadol no } \\
\text { prolongamento do TLEl é significativamente superior (MD 1,21; } \\
\text { IC95\%: } 0,23-2,17 ; P=0,02 \text { ) }\end{array}$ & 2 \\
\hline
\end{tabular}

Legenda: $\mathrm{EAC}=$ Ensaio aleatorizado controlado; $\mathrm{ECNA=Ensaio} \mathrm{clínico} \mathrm{não} \mathrm{aleatorizado;} T L E l=T e m p o$ de latência ejaculatória intravaginal; $\mathrm{EP}=$ Ejaculação prematura; $S M D=S$ tandard mean difference; $I C=$ Intervalo de confiança; OR=Odds ratio; $R R=$ Risco relativo; $N E=$ Nível de evidência. 


\begin{tabular}{|c|c|c|c|c|}
\hline Referência & Estudos incluídos & Intervenção & Resultados & NE \\
\hline $\begin{array}{l}\text { Kirby EW, et al } \\
(2015) \\
\text { EUA }\end{array}$ & $\begin{array}{l}8 \text { Ensaios clínicos } \\
\text { (4 EAC + } 4 \text { ENAC) } \\
\text { Follow-up } 3 \text { a } 24 \\
\text { semanas }\end{array}$ & $\begin{array}{l}\text { Tramadol vs. Placebo } \\
\text { Outcomes: Eficácia e } \\
\text { segurança } \\
\text { (4 EAC + } 3 \text { ENAC) } \\
\text { Tramadol vs. Paroxetina } \\
\text { Outcomes: Eficácia } \\
\text { (2 ENAC) }\end{array}$ & $\begin{array}{l}\text { Em } 7 \text { ensaios clínicos verificou-se um prolongamento do } \\
\text { TLEI relativamente ao placebo. } \\
\text { Dos } 8 \text { ensaios clínicos, } 7 \text { avaliaram os efeitos adversos do } \\
\text { tramadol. Em geral, o tramadol foi bem tolerado, sendo } \\
\text { os efeitos adversos mais frequentes a sonolência e } \\
\text { efeitos gastrointestinais superiores } \\
\text { Um ensaio clínico revelou que o tramadol conduz a um } \\
\text { prolongamento do IELT superior à paroxetina ( } 6,33 \text { min } \\
\text { vs. } 2,23 \text { seg) } \\
\text { Um ensaio clínico revelou um prolongamento do TLEI } \\
\text { superior quando se usa a paroxetina relativamente ao } \\
\text { Tramadol (180 seg vs. } 110 \text { seg) }\end{array}$ & 2 \\
\hline
\end{tabular}

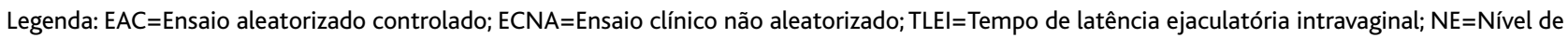
evidência.

do à elevada heterogeneidade dos estudos avaliados $\left(I^{2}=97 \%\right)$, pelo que o seu potencial efeito benéfico deve ser cuidadosamente avaliado em comparação com o risco de dependência. O risco de adição é elevado, especialmente naqueles indivíduos com relações sexuais frequentes.

No mesmo ano foi publicada a MA de James e colaboradores (Quadro II) que incluiu oito ensaios clíni$\cos ^{20}$ Na maioria dos estudos, a amostra foi constituída por homens com EP primária, sem disfunção erétil e, em todos os estudos, o tramadol foi prescrito, on-demand, em dose compreendida entre 25 e $100 \mathrm{mg}$, uma a quatro horas antes da relação sexual. O follow-up variou entre quatro a seis meses, tendo a maioria dos estudos terminado oito semanas após o tratamento. Para avaliar a eficácia, quatro ensaios clínicos compararam o tramadol versus placebo e concluíram que o tramadol é significativamente mais efetivo, havendo uma diferença média de 1,24 minutos do TLEI após oito a 12 semanas de tratamento. Contudo, é evidente a elevada heterogeneidade entre os estudos $\left(I^{2}=74 \%\right)$. Verificou-se também que o efeito do tramadol no prolongamento do TLEI é superior ao efeito do sildenafil, da terapia comportamental e da lidocaína tópica. Para avaliar a segurança, cinco ensaios clínicos (total de 583 indivíduos), com baixa heterogeneidade entre si $\left(\mathrm{I}^{2}=0 \%\right)$, compararam a frequência dos efeitos adversos do tramadol com o placebo e verificaram que o tramadol apresenta significativamente maior risco de efeitos adversos. Quando comparado com a terapia comportamental isolada, um ensaio clínico (total de 72 indivíduos) concluiu também que os efeitos adversos são significativamente mais frequentes com o tramadol.

A RS de Kirby e colaboradores, publicada em 2015, incluiu oito ensaios clínicos que avaliaram separadamente tramadol versus placebo e tramadol versus paroxetina. ${ }^{21}$ A duração do tratamento nos diferentes estudos variou entre três a 24 semanas e a dose entre $25 \mathrm{mg}$ a $100 \mathrm{mg}$. Foi avaliado o uso de tramadol, on-demand e diariamente, no tratamento da EP. Sete estudos compararam tramadol versus placebo e em todos verificou-se um aumento do TLEI relativamente ao placebo. Embora cada um dos estudos incluídos tenha mostrado aumento no TLEI, houve uma grande variabilidade no grau de resposta entre os estudos (o prolongamento do TLEI entre os indivíduos tratados com a dose de $25 \mathrm{mg}$ variou entre 116 segundos num estudo e mais do que 10 minutos noutro). Dois estudos compararam tramadol versus paroxetina. Embora tenham obtido resultados contraditórios, tiveram diferenças consideráveis no desenho dos estudos - um dos estudos demonstrou superioridade da paroxetina, avaliada numa 
toma diária, enquanto o outro mostrou superioridade do tramadol, avaliando a toma de paroxetina on-demand. Todos os estudos incluídos, à exceção de um, avaliaram os efeitos adversos. Em geral, o tramadol foi bem tolerado. Os efeitos adversos mais comuns foram a sonolência e os efeitos gastrointestinais superiores (náuseas, dispepsia e vómitos), com uma incidência global que ronda os 10-30\%. Estes efeitos laterais são mais frequentes e mais severos com doses mais elevadas. Este estudo demonstrou que, embora o risco de dependência e abuso não seja negligenciável, é extremamente baixo.

\section{CONCLUSÃO}

Perante a evidência disponível, esta revisão permitiu-nos concluir que a utilização do tramadol no tratamento da EP apresenta evidência limitada (FR B). A utilização de diferentes metodologias contribuiu para a heterogeneidade dos estudos até agora efetuados, diminuindo a firmeza das conclusões obtidas.

As diferenças encontradas entre os estudos podem ser explicadas pelas limitações que são transversais, na generalidade, a todos os artigos incluídos. Houve variabilidade entre os estudos relativamente à definição de EP - alguns estudos utilizaram a definição mais ampla da Diagnostic and Statistical Manual of Mental Disorders, $4^{\text {th }}$ ed., text revision (DSM-IV-TR), outros estudos utilizaram a definição mais estrita da ISSM e alguns estudos não forneceram qualquer definição. Embora o TLEI seja um dado objetivo fundamental na avaliação do doente, a sua determinação na prática clínica é difícil. Além disso, há doentes que têm a sensação que são ejaculadores prematuros, o que pode causar angústia e frustração, apesar de terem um TLEI muito acima do considerado patológico. Adicionalmente, a maioria dos estudos não apresentou uniformização nos critérios de inclusão, nomeadamente nem todos os indivíduos foram especificamente descritos como tendo EP primária ou secundária. Devido ao facto destes dois processos resultarem de diferentes mecanismos, não é claro se a resposta ao tratamento poderia ser diferente entre estes grupos. Por fim, a inconsistência nas doses de tramadol utilizadas nos diferentes estudos, bem como a curta duração de follow-up na maioria dos estudos, dificulta a avaliação do efeito do tramadol a longo prazo, relativamente aos efeitos laterais e risco de dependência, principalmente naqueles indivíduos com história de adição de narcóticos ou abuso de drogas.

Serão necessários estudos futuros de elevada qualidade metodológica, envolvendo um maior número de indivíduos e com uniformização dos critérios de inclusão e dos outcomes avaliados, de forma a obter conclusões firmes sobre a eficácia e segurança do uso de tramadol a longo prazo no tratamento da EP.

\section{REFERÊNCIAS BIBLIOGRÁFICAS}

1. Bejma JP, Hellstrom WJ. Premature ejaculation. Am Urol Assoc Update Series. 2007;26:365-71.

2. Porst H, Montorsi F, Rosen RC, Gaynor L, Grupe S, Alexander J. The Premature Ejaculation Prevalence and Attitudes (PEPA) survey: prevalence, comorbidities, and professional help-seeking. Eur Urol. 2007;51(3):816-24.

3. McMahon CG, Althof SE, Waldinger MD, Porst H, Dean J, Sharlip ID, et al. An evidence-based definition of lifelong premature ejaculation: report of the International Society for Sexual Medicine (ISMM) ad hoc committee for the definition of premature ejaculation. J Sex Med. 2008;5(7):1590-606.

4. Serefoglu EC, McMahon CG, Waldinger MD, Althof SE, Shindel A, Adaikan $\mathrm{G}$, et al. An evidence-based unified definition of lifeling and acquired premature ejaculation: report of the second International Society for Sexual Medicine ad hoc committee for the definition of premature ejaculation. J Sex Med. 2014;11(6):1423-41.

5. Morales A, Barada J, Wyllie MG. A review of the current status of topical treatments for premature ejaculation. BJU Int. 2007;100(3): 493-501.

6. Richardson D, Goldmeier D. Premature ejaculation: does country of origin tell us anything about etiology? J Sex Med. 2005;2(4):508-12.

7. McMahon CG, Abdo C, Incrocci L, Perelman M, Rowland D, Waldinger $M$, et al. Disorders of orgasm and ejaculation in men. J Sex Med. 2004; 1(1):58-65.

8. Carvalho AP. Urologia em medicina familiar: ejaculação prematura. Lisboa: Associação Portuguesa de Urologia; 2014.

9. Bar-Or D, Salottolo KM, Orlando A, Winkler JV. A randomized doubleblind, placebo-controlled multicenter study to evaluate the efficacy and safety of two doses of the tramadol orally disintegrating tablet for the treatment of premature ejaculation within less than 2 minutes. Eur Urol. 2012;61(4):736-43.

10. Kaynar M, Kilic O, Yurdakul T. On-demand tramadol hydrochloride use in premature ejaculation treatment. Urology. 2012;79(1):145-9.

11. Marcou TA, Marque S, Mazoit JX, Benhamou D. The median effective dose of tramadol and morphine for postoperative patients: a study of interactions. Anesth Analg. 2005;100(2):469-74.

12. Safarinejad MR, Hosseini SY. Safety and efficacy of tramadol in the treatment of premature ejaculation in the treatment of premature ejaculation: a double-blind, placebo-controlled, fixed-dose,randomized study. J Clin Psychopharmacol. 2006;26(1):27-31.

13. Salem EA, Wilson SK, Bissada NK, Delk JR, Hellstrom WJ, Cleves MA. Tramadol $\mathrm{HCL}$ has promise in on-demand use to treat premature ejaculation. J Sex Med 2008;5(1):188-93. 
14. Alghobary M, El-Bayoumy Y, MostafaY, Mahmound el-HM,Amr M. Evaluation of tramadol on demand vs. daily paroxetine as a long-term treatment of lifelong premature ejaculation. J Sex Med. 2010;7(8): 2860-7.

15. Althof SE, Abdo CH, Dean J, Hackett G, McCabe M, McMahon CG, et al. International Society for Sexual Medicine's Guidelines for the Diagnosis and Treatment of Premature Ejaculation. J Sex Med. 2010;7(9): 2947-69.

16. Hatzimouratidis K, Amar E, Eardley I, Giuliano F, Hatzichristou D, Montorsi $F$, et al. Guidelines on male sexual dysfunction: erectile dysfunction and premature ejaculation. Eur Urol. 2010;57(5):804-14.

17. Wu T, Yue X, Duan X, Luo D, Cheng Y, Tian Y, et al. Efficacy and safety of tramadol for premature ejaculation: a systematic review and metaanalysis. Urology. 2012;80(3):618-24.

18. Yang L, Qian S, Liu H, Liu L, Pu C, Han P, et al. Role of tramadol in premature ejaculation: a systematic review and meta-analysis. Urol Int. 2013;91(2):197-205.

19. Castiglione F, Albersen M, Hedlund P, Gratzke C, Salonia A, Giuliano F. Current pharmacological management of premature ejaculation: a systematic review and meta-analysis. Eur Urol. 2016;69(5):904-16.
20. Martyn-St James M, Cooper K, Kaltenthaler E, Dickinson K, Cantrell A, Wylie K, et al. Tramadol for premature ejaculation: a systematic review and meta-analysis. BMC Urol. 2015;15:6.

21. Kirby EW, Carson CC, Coward RM. Tramadol for the management of premature ejaculation: a timely systematic review. Int J Impot Res. 2015;27(4):121-7.

\section{CONFLITOS DE INTERESSE}

Os autores declaram não ter quaisquer conflitos de interesse.

\section{FINANCIAMENTO DO ESTUDO}

Este artigo não foi objeto de qualquer tipo de financiamento.

\section{ENDEREÇO PARA CORRESPONDÊNCIA}

Isabel Machado

E-mail: isabelmachado8@hotmail.com

Recebido em 02-06-2016

Aceite para publicação em 26-11-2017

\section{ABSTRACT}

\section{EFFICACY AND SAFETY OF TRAMADOL IN PATIENTS WITH PREMATURE EJACULATION: EVIDENCE BASED REVIEW}

Objective: Premature ejaculation (PE) is the most prevalent male sexual dysfunction, affecting $20-30 \%$ of all men. In recent years, many pharmacological alternatives have been used to treat PE. One of the treatments that has recently proved to be promising in the treatment of PE is on demand use of tramadol. However, some studies have showed inconsistent results regarding its use. Thus, the aim of this study was to review the most current evidence on the efficacy and safety of tramadol in the treatment of PE.

Data sources: National Guideline Clearing House, NICE, Canadian Medical Association Practice Guidelines, DARE, Cochrane, PubMed and TRIP Database.

Methods: We searched for meta-analyses systematic reviews, observational studies and clinical guidelines / evidence-based guidelines published in Portuguese, Spanish and English, using the MeSH terms 'tramadol' and 'premature ejaculation'. The Strength of Recommendation Taxonomy (SORT) scale of the American Academy of Family Physicians was used to assign levels of evidence and strength of recommendations.

Results: Fifty six articles were found and, amongst these, seven met the inclusion criteria: two clinical practice guidelines, four meta-analyses, and one systematic review. Most studies showed that the use of tramadol is effective when compared to placebo, but not when compared with the use of paroxetine. However, the use of different methodologies contributed to the heterogeneity of the results, reducing the strength of the conclusions obtained.

Conclusion: According to the evidence, this review allowed us to conclude that the use of tramadol in the treatment of PE has limited evidence (SORT B). Further studies of high quality, including a greater number of individuals with homogenous inclusion criteria and outcomes are needed in order to obtain stronger conclusions about the long term use of tramadol in the treatment of PE.

Keywords: Tramadol; Premature ejaculation 